\title{
Ultrahigh vacuum chamber for synchrotron x-ray diffraction from films adsorbed on single-crystal surfaces
}

\author{
J. R. Dennison \\ Department of Physics, Utah State University, Logan, Utah 84322 \\ S.-K. Wang, P. Dai, T. Angot, and H. Taub \\ Department of Physics and Astronomy, University of Missouri-Columbia, Columbia, Missouri 65211 \\ S. N. Ehrlicha) \\ School of Materials Engineering, Purdue University, West Lafayette, Indiana 47907
}

(Received 20 January 1992; accepted for publication 8 April 1992)

\begin{abstract}
An ultrahigh vacuum chamber has been developed for structural analysis of adsorbed films and single-crystal surfaces using synchrotron $x$-ray diffraction. It is particularly well suited for investigations of physisorbed and other weakly bound films. The chamber is small enough to transport and mount directly on a standard four-axis diffractometer and can also be used independently of the $\mathrm{x}$-ray diffractometer. A low-current, pulse-counting, low-energy electron diffraction/Auger spectroscopy system with a position-sensitive detector enables in situ characterization of the film and substrate while the sample is located at the $\mathrm{x}$-ray scattering position. A closed-cycle $\mathrm{He}$ refrigerator and electron bombardment heater provide controlled substrate temperatures from 30 to $1300 \mathrm{~K}$. The chamber is also equipped with an ion sputter gun, a quadrupole mass spectrometer, and a gas handling system. Details of the design and operation of the instrument are described. To demonstrate the performance of the instrument, we present some preliminary results of a study of Xe physisorbed on the $\mathrm{Ag}(111)$ surface.
\end{abstract}

\section{INTRODUCTION}

Over the past decade, $\mathrm{x}$-ray diffraction using synchrotron radiation has developed rapidly as a technique for characterizing the structure of surfaces, interfaces, and adsorbed films. As discussed in a recent review, ${ }^{1}$ the technique does not suffer from the multiple scattering effects which complicate the analysis of charged-particle diffraction from surfaces, while it offers exceptionally high resolution in reciprocal space and sufficient sensitivity to probe a single layer of atoms.

A number of ultrahigh vacuum (UHV) chambers have been built in recent years for use in surface $x$-ray diffraction experiments at synchrotron sources. ${ }^{2-5}$ These chambers share the common features of allowing $\mathrm{x}$ rays access to the sample through a beryllium window and in providing the six degrees of freedom for rotating (at least over some limited range) and translating the sample in the $\mathrm{x}$-ray beam. The difficulty of rotating the sample precisely while maintaining it under UHV conditions and the need to characterize its surface by various auxiliary probes have motivated a variety of chamber designs. These differ in whether the sample rotations are accomplished within the chamber or by rotating the entire chamber, in the number and proximity of various auxiliary surface analytical probes, in the sample preparation capabilities, and in the number of degrees of freedom provided for detector motion. A summary of various approaches to chamber design has been given in Feidenhans'l's review. ${ }^{1}$

Clearly, the type of sample to be investigated plays a

\footnotetext{
'Present address: NSLS, Brookhaven National Laboratory, Upton, New York 11973.
}

decisive role in dictating the chamber design. The primary purpose of the chamber described here was the study of physisorbed and other weakly bound films on single-crystal substrates. These experiments have several basic requirements: (1) an extensive sample characterization process which is performed most efficiently while the sample is out of the x-ray beam; (2) a method independent of x-ray diffraction to characterize the film structure, its thickness, and the substrate quality while the sample is mounted in the x-ray beam; (3) a low-temperature capability; and (4) a method of cleaning the sample within the chamber.

The above experimental requirements resulted in a number of features which have not been combined in previous chamber designs. In order to achieve the sample characterization capability out of the x-ray beam, we chose to make our chamber small enough to transport easily. Its compact design allows mounting on a general-purpose four-circle diffractometer (see Fig. 1) used at many synchrotron beam lines such as those at the National Synchrotron Light Source (NSLS) at Brookhaven National Laboratory. Our design was adapted from one developed earlier by Zschack et al. ${ }^{5,6}$ for use at the X18A beam line of the MATRIX $^{7}$ Participating Research Team at the NSLS. We note that the small chamber size also results in a cost substantially less than larger chambers requiring dedicated diffractometers.

Despitc its small size, our chamber has several capabilities not found in the transfer cells or "baby chambers" originally used in surface $x$-ray diffraction. ${ }^{8}$ It contains a low-energy electron diffraction (LEED) system which allows in situ characterization of physisorbed films and the single-crystal substrate. As in the chamber of Vlieg et al., LEED and Auger spectroscopy can be performed without 
moving the sample from the $\mathrm{x}$-ray scattering position; however, in our chamber this is accomplished without moving these analysis probes. Our LEED system has a commercially available pulse-counting, position-sensitive detector requiring a very low incident electron-beam current. The low beam current is useful in minimizing desorption of weakly bound films.

A feature of our chamber which has not appeared in previous designs ${ }^{2-5}$ is a low-temperature capability. Using a commercially available cryogenic refrigerator, a sample temperature down to $30 \mathrm{~K}$ can be achieved. Such temperatures are necessary to condense some physisorbed films or to reach phase transitions which they exhibit. In addition, temperatures as high as $1300 \mathrm{~K}$ for cleaning and annealing the sample can be attained with an electron-bombardment heater.

This paper is organized as follows. In Sec. II, we describe the mounting of the chamber on the diffractometer, the sample alignment, and the scattering geometry used in the surface diffraction experiments. This description includes the range of motions accessible to the sample (and chamber) and the resulting volume in reciprocal space available in a typical experiment. Section III discusses details of the chamber's interior components with emphasis on the sample stage and the LEED/Auger system. In Sec. IV, we present results of some preliminary experiments with xenon adsorbed on a $\mathrm{Ag}$ (111) surface which demonstrate the capabilities of the chamber for the investigation of physisorbed films. The paper concludes with a capsule summary in Sec. V.

\section{CHAMBER MOUNTING, SAMPLE ALIGNMENT, AND SCATTERING GEOMETRY}

Our chamber was designed to mount on the Huber Model 5020 diffractometer at the MATRIX beam line of the NSLS. The diffractometer was not modified in any way in order to accept the $120 \mathrm{~kg}$ chamber. With minor changes to the chamber such as different counterweights, it could be mounted on any similar diffractometer of comparable size.

The diffractometer has four circles labeled $\phi, \chi, \omega$, and $2 \theta^{\prime}$ as shown schematically in Fig. 1(a). We use $2 \theta^{\prime}$ to specify the position of the detector arm as distinguished from $2 \theta$, the scattering angle between the incident and scattered wave vectors, $\mathbf{k}_{\mathbf{i}}$ and $\mathbf{k}_{\mathbf{f}}$, respectively. The two support points of the chamber are the upper and lower $\phi$ mounts of the 50-cm-diam $\chi$ circle, $\mathrm{H} 5$ and $\mathrm{H} 6$, respectively, in Fig. 2. To minimize the gravitational torque on the diffractometer gears, two counterweights having a combined mass of $\sim 85 \mathrm{~kg}$ are required, one in front of and the other behind the $\chi$ circle. Using standard computer control of the diffractometer, the chamber can be rotated by the $\chi$ and $\omega$ drives with an angular resolution of $0.001^{\circ}$. The smaller motor on the $\phi$ drive requires a 10:1 gear reducer to move the chamber with an angular resolution of $0.005^{\circ}$.

The mounting procedure is facilitated by transferring the chamber to a bench, fixed to the same table on which the diffractometer resides, which positions the bottom of

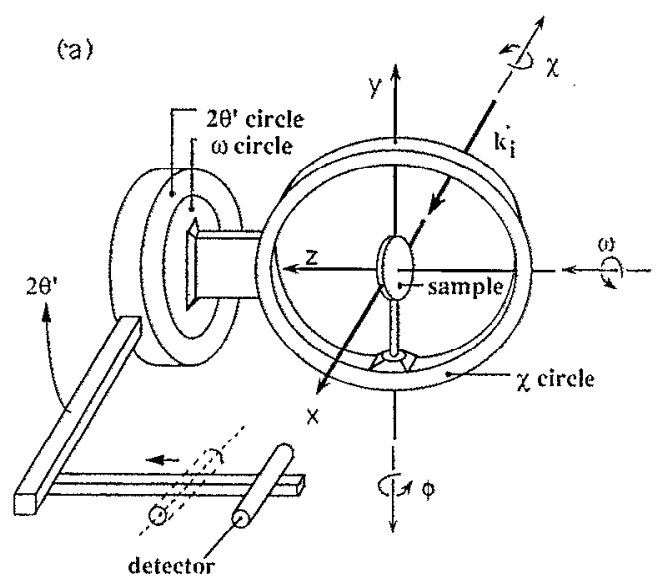

(b)

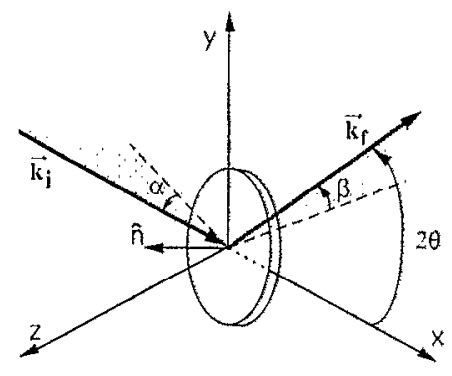

FIG. 1. (a) Schematic diagram of a four-circle diffractometer after $R$. Feidenhans'l (Ref. 1), Diffractometer and sample are in their unrotated positions $\left(\phi=\chi=\omega=2 \theta^{\prime}=0\right)$. The dashed detector represents a modification allowing for motion of the detector out of the scatiering plane; the direction of its linear motion is shown by the arrow. (b) Real-space scattering geometry: the incident beam of wave vector $\mathrm{k}_{\mathrm{i}}$ is directed along the $+x$ axis and makes an angle $2 \theta$ with the outgoing wave vector $\mathrm{k}_{f} ; \alpha$ and $\beta$ are the angles of the incident and diffracted beam, respectively, with the sample surface.

the chamber support frame at the same height as the lower $\phi$ mount. Time to mount or dismount the chamber is $\sim 4$ $\mathrm{h}$. This includes installing the counterweights, securing electrical cables, and connecting gas supply and return lines between the compressor and the cryostat head.

Once the chamber is mounted on the Huber $\chi$ circle, the sample surface can be centered in the incident $x$-ray beam and its orientation determined. To benefit from the strong polarization of the synchrotron radiation in the horizontal plane, the detector $\left(2 \theta^{\prime} \mathrm{arm}\right)$ of the standard diffractometer moves in a vertical plane. Thus, for grazingangle diffraction, the sample is initially mounted in the chamber with the unit normal $\hat{n}$ of the surface of interest directed along the $+z$ axis of Fig. 1. The x-ray beam enters and exits the chamber through a cylindrical Be window with $360^{\circ}$ access [labeled B in Figs. 2(a) and 3]. The window has a $10 \mathrm{~cm}$ diameter, a $2.5 \mathrm{~cm}$ length, and a wall thickness of $0.04 \mathrm{~cm} .{ }^{9}$ Centering of the sample surface in the incident beam is accomplished with the three translational degrees of freedom of the manipulator shown in Fig. 2(a).

The orientation of the surface normal can be determined by specular reflection of the $x$-ray beam at angles 
(a)

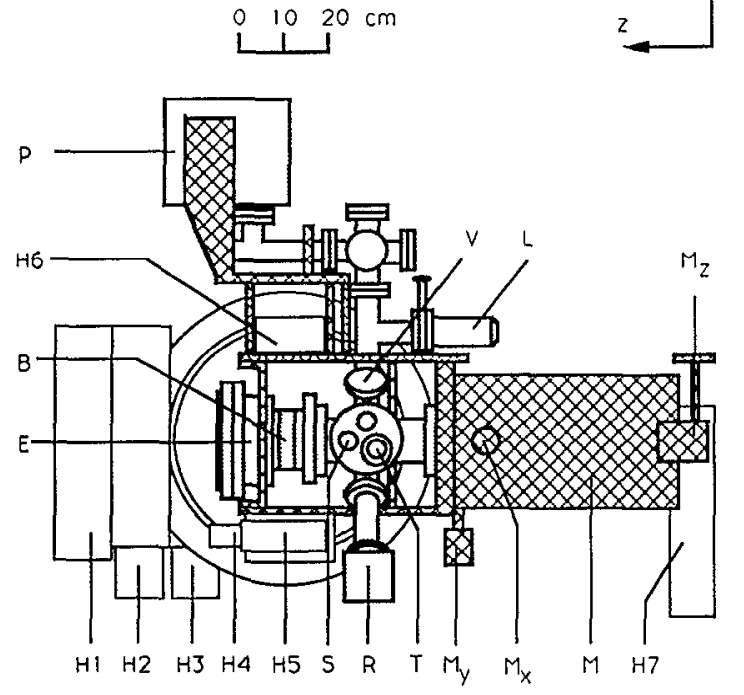

(b)

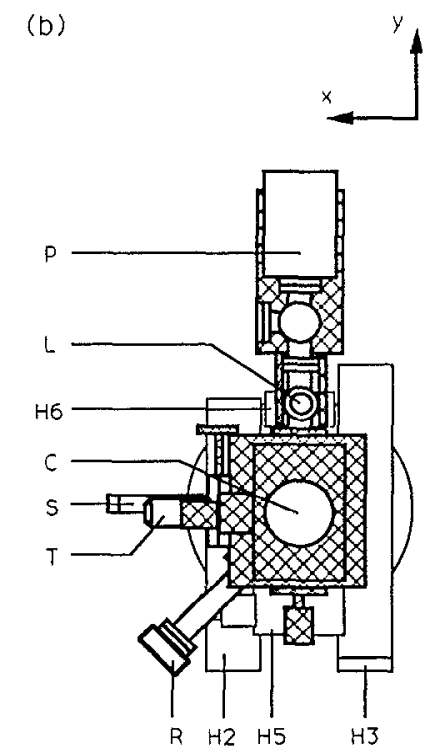

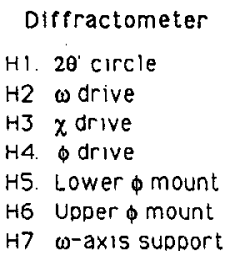

Chamber

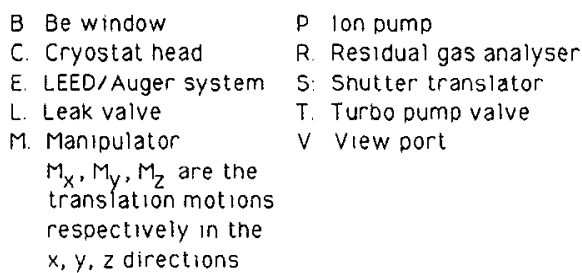

FIG. 2. Schematic diagram of the UHV chamber mounted on the diffractometer in its unrotated position $(\phi=\chi=\omega=0)$. Axes are defined as in Fig. 1 . Hatched sections indicate the manipulator and support frame; chamber parts are shaded. (a) View looking toward the synchrotron source along the $-x$ axis. (b) View from the cryostat head along the $+z$ axis. For clarity the $\omega$ axis support $\mathrm{H} 7$ has been omitted.

$\leqslant 1^{\circ}$. This bound on the reflection angle is imposed in the present design by a limit of $1.5^{\circ}$ motion in the negative $\phi$ direction (the range is $21^{\circ}$ in the positive $\phi$ direction). A modification of the detector motion which will extend the range of the reflection angle is now being implemented as will be discussed below. In our experiments thus far, we have oriented the sample crystallographically using three bulk reflections of the substrate. The miscut of the surface was checked before mounting the sample in the chamber using reflection of laser light to measure the angle between the surface normal and the previously determined crystallographic axes.

During a diffraction scan, the sample (along with the rest of the chamber) is rotated by the $\phi, \chi$, and $\omega$ drives while the detector moves in $2 \theta^{\prime}$ so as to determine the momentum transfer parallel and perpendicular to the surface, $\mathbf{Q}_{\|}$and $\mathbf{Q}_{1}$, respectively. Since only three independent rotational motions are required to specify $\mathbf{Q}$, an extra degree of freedom is available so that a scan may be done at fixed incident angle $\alpha$ or with $\alpha$ equal to the exit angle $\beta$ [see Fig. 1(b)]. Computer codes to calculate the angle settings are available. ${ }^{10,11}$

The volume of reciprocal space accessible in a surface diffraction experiment is determined by the size of the chamber's Be window, the constraints imposed on the chamber motion by diffractometer components, and the obstruction of the incident $x$-ray beam by the $\chi$ circle of the diffractometer. For example, a typical in-plane radial scan using $1.5 \AA$ x rays would have a $Q_{||}$range of $0.5-4.8 \AA^{-1}$ at a fixed $Q_{1}=0.2 \AA^{-1}$ and a fixed incident angle $\alpha=1.4^{\circ}$. The $Q_{\|}$range in this case is determined by the limits of the $\phi$ motion. The limit on the perpendicular momentum transfer $Q_{1}$ depends on the value of $Q_{\|}$. For $Q_{\|}=1.65 \AA^{-1}$ [the value for the $\mathrm{Xe}(10)$ rod in our experiments (see Sec. IV)], the maximum value of $Q_{1}$ is $1.3 \AA^{-1}$ as determined by the accessible range of the $\chi$ motion, $-2^{\circ} \leqslant \chi \leqslant 38^{\circ}$.

In order to increase the volume of reciprocal space accessible with our chamber, we are planning two modifcations of our system. First, a new sample holder is being constructed which will allow rotation of the sample within the chamber about the surface normal. This will permit us to study symmetry equivalent reflections of the sample whose accessibility is now limited by the range of the $\omega$ motion, $-2^{\circ} \leqslant \omega \leqslant 48^{\circ}$. The second modification is to add a linear drive to the detector arm so that the detector can move out of the scattering plane [see dashed detector in Fig. 1(a)]. The so-called extended four-circle diffractometer ${ }^{10,11}$ offers some of the advantages of the five-circle instrument used by Vlieg et al., ${ }^{4,12}$ particularly the increase in the maximum $Q_{1}$ accessible in both specular and nonspecular rod scans. 

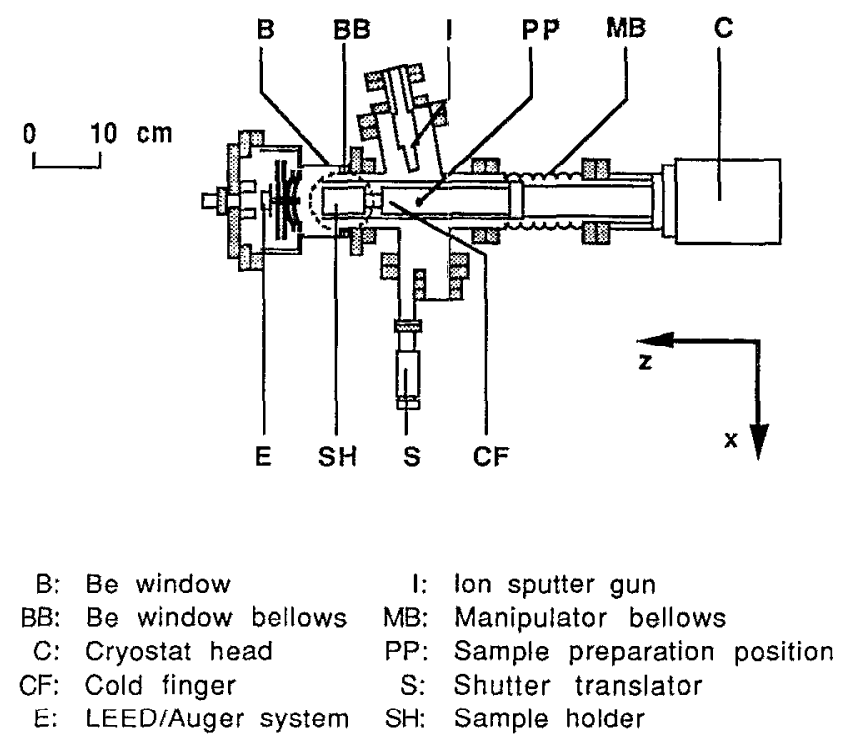

FIG. 3. Top view of the interior chamber components. The sample is at the $x$-ray/LEED position; the sample preparation position is identified by PP.

\section{CHAMBER COMPONENTS}

A top view of the chamber's principal interior components is given schematically in Fig. 3 . The sample is shown at the $\mathrm{x}$-ray scattering position which coincides with the focus of the LEED/Auger optics. It can be withdrawn to the sample preparation position (PP) for sputtering and annealing. This section describes in more detail some of the interior components.

\section{A. The sample stage and manipulator}

Figure 4 presents a detailed view of the sample stage. The sample (SS) is a single-crystal disk $(0.3 \mathrm{~cm}$ thick by $1.2 \mathrm{~cm}$ diameter). A small relief which is electron are cut on its surface allows the sample to be firmly held by a clamp (SC) for good thermal contact with the sample holder (SH). The sample stage is mounted rigidly on the cold finger (CF) of a closed-cycle He refrigerator (APD Cryogenics) which can cool the sample to $<30 \mathrm{~K}$ as measured by a Au-chromel thermocouple (TC). A radiation shield (RS) extends from the cryostat first stage to the sample.

The sample stage is constructed of refractory materials ( $\mathrm{Ta}, \mathrm{Ti}$, and $\mathrm{W}$ ) in order to withstand the high temperatures required for surface cleaning and annealing. With the sample at the preparation position (PP in Fig. 3), an electron bombardment heater (HF in Fig. 4) located behind the sample allows temperatures up to $1300 \mathrm{~K}$ to be reached as monitored by a chromel-alumel thermocouple (not shown in Fig. 4).

The substrate is cleaned by a combination of ion sputtering ( $\mathrm{I}$ in Fig. 3 ) and thermal desorption. Hightemperature annealing is used to remove sputtering damage to the substrate. The sample can be inspected visually through a 4-cm-diam Pyrex viewport [V in Fig. 2(a)]. A shutter ( $\mathrm{S}$ in Figs. 2 and 3 ) isolates the LEED optics and Be window from the preparation compartment to prevent
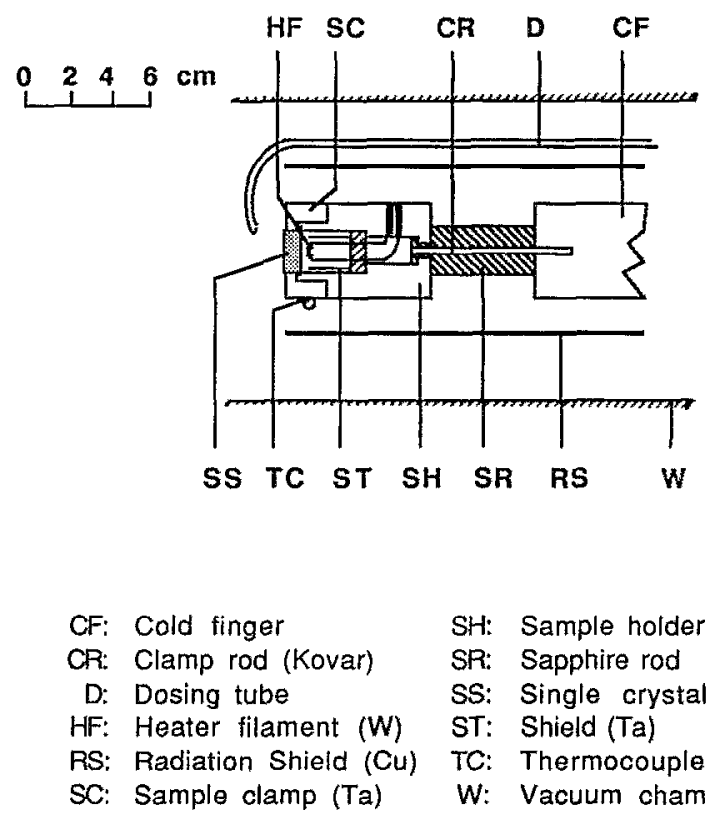

SH: Sample holder (Ti)

SR: Sapphire rod

SS: Single crystal sample

ST: Shield (Ta)

TC: Thermocouple

W: Vacuum chamber wall

FIG. 4. Sample stage: magnification of the region enclosed by the dashed circle in Fig. 3 showing the sample holder elements.

their damage during sputtering and heating. A thin annuular cover plate (not shown in Fig. 4) of the same material as the sample is used to cover the sample clamp (SC). This reduces contamination of the sample during sputtering and annealing.

To avoid damage to the cryostat cold stage during heating, the sample stage is isolated from the cold finger by a single-crystal sapphire rod $5 \mathrm{~cm}$ long and $2.5 \mathrm{~cm}$ diameter (SR in Fig. 4). The sapphire acts as a thermal switch, providing good thermal insulation at high temperatures and high thermal conductivity at low temperatures. The cryostat is operated when heating the sample above room temperature. At sample temperatures of $1000 \mathrm{~K}$ the cold stage is at $<100 \mathrm{~K}$. The sapphire rod also electrically isolates the sample stage, facilitating sample current measurements.

The sample can be retracted from the LEED/Auger/ $\mathrm{x}$-ray position to the sample preparation position ( $\mathrm{PP}$ in Fig. 3) while the chamber is mounted on the diffractometer. Three independent translational motions are provided by the manipulator [M in Fig. 2(a)] to which the cryostat head is attached. A stepping motor drives the $z$ axis motion $\left[\mathrm{M}_{\mathrm{z}}\right.$ in Fig. 2(a)] which provides the $15 \mathrm{~cm}$ of travel between the $x$-ray and preparation positions and allows precision centering of the sample in the $\mathrm{x}$-ray beam. The $x$ and $y$ motions of the manipulator, which are also used for centering the sample in the $x$-ray beam, have a range of $\pm 1 \mathrm{~cm}$ and are operated manually with micrometers.

\section{B. Pumping and gas dosing}

The entire chamber is usually baked at $\sim 100{ }^{\circ} \mathrm{C}$. This temperature limit is imposed to avoid having to remove the phenolic displacer within the cryostat. Higher bake-out 
temperatures are possible, if time and care are taken to remove the displacer. After a $100^{\circ} \mathrm{C}$ bakeout, the base pressure in the chamber is $\sim 1 \times 10^{-9}$ Torr at room temperature which reduces to a pressure of $<5 \times 10^{-10}$ Torr when the cryostat is operating. Pumping is provided by a $25 \mathrm{l} / \mathrm{s}$ triode ion pump ( $\mathrm{P}$ in Fig. 2) and a $50 \mathrm{l} / \mathrm{s}$ turbo pump (Balzers) mounted on the chamber through an isolation valve ( $\mathrm{T}$ in Fig. 2). A titanium sublimation pump can also be used. There is a Dycor model M100 residual gas analyzer ( $\mathrm{R}$ in Fig. 2 ) used primarily for leak detection and monitoring chamber cleanliness.

Gases for adsorption can be admitted to the chamber in two ways. For equilibrium adsorption, the entire chamber can be backfilled through an UHV leak valve ( $\mathrm{L}$ in Fig. 2). Alternatively, the substrate is exposed to gas through a dosing tube ( $D$ in Fig. 4, located $1.0 \mathrm{~cm}$ from the substrate) while the cryostat and turbopump are operating. This technique was used by Unguris et al. ${ }^{13}$ in LEED studies of rare gases adsorbed on single-crystal substrates. Since this is not a true thermal equilibrium situation, an independent method of coverage determination must be employed, e.g., by monitoring the attenuation of a LEED beam diffracted from the substrate.

\section{LEED/Auger system}

The ability to perform in situ characterization of the substrate surface and adsorbed films is essential for physisorption experiments in which surface degradation is rapid. Our primary characterization tool is a LEED/ Auger system (see Fig. 3) based on the design of Stair. ${ }^{14}$ The large diameter Be window allows the focal point of the LEED optics to be at the x-ray sample position (SS in Fig. 4). In this way, $x$ ray, LEED, and Auger measurements are possible without moving either the sample or the surface analysis probes. Due to the sensitivity of the electron multiplier in the LEED detector to photoelectrons produced by the $x$-ray beam, it is not possible to use the LEED system while the $\mathrm{x}$-ray beam is on. However, as described below, the LEED and $x$-ray measurements can be done alternately with sufficient speed that the LEED system has proved to be a convenient means of characterizing the substrate and film just prior to and during the $x$-ray experiment.

Since the chamber is also usable in a stand-alone configuration, the LEED/Auger system allows a survey of film structures at various coverages and temperatures away from the synchrotron source. This "dress rehearsal" feature enables a more efficient use of the synchrotron beam time.

The main reason for selecting Stair's design for the LEED system was its compactness compared to other lowcurrent LEED systems. ${ }^{15}$ By utilizing a position-sensitive, pulse-counting electron detector, there is no need for the chamber to include a phosphor screen, mirror, viewport, and camera required for photographic or video recording. The LEED optics and Be window are attached to the main chamber via an $11 \mathrm{~cm}$ i.d. stainless-steel bellows (BB in Fig. 3) which serves to minimize strain on the thin $B e$ window. A set of gimbal screws fix the orientation of the
LEED optics flange with respect to the main chamber. The bellows allows $\sim \pm 0.4 \mathrm{~cm}$ translation and $\pm 1^{\circ}$ polar rotation of the incident electron beam with respect to the surface normal.

The LEED/Auger detector system (Fig. 3) uses a 7.5cm-diam electron-multiplier chevron array (Galileo Electro-Optics) to create an electron pulse incident on a flat resistive anode detector [Surface Science Laboratories (now Quantar Technology)]. A very low incident beam current $(\sim 10 \mathrm{pA})$ prevents saturation of the detector. For Auger spectroscopy, the electron-multiplier gain is reduced to near unity and incident beam currents of 2-3 $\mu \mathrm{A}$ are used. A position computer (Surface Science model 2401) typically allows a count rate of $30 \mathrm{kHz}$ with a maximum LEED peak-to-background ratio of $\sim 40$. A standard electron gun (Perkin-Elmer) used for both LEED and Auger spectroscopy is mounted behind the detector. Colinear holes in the detector, electron-multiplier channel plates, and grids accommodate the electron gun drift tube. The entire LEED optics is surrounded by a $\mu$-metal shield to reduce magnetic fields and electronic noise. A mask was temporarily mounted in front of the electron multiplier for calibration of the detector and check of its linearity.

The electronically recorded LEED pattern is displayed in real time on an oscilloscope. Using a commercially available interface and software (Quantar Technology), the LEED pattern can be integrated over a preset accumulation time and stored digitally in a personal computer (IBM AT) for further analysis. The Auger spectra are also digitized through an interface (National Instruments) and stored in the same computer.

\section{PRELIMINARY MEASUREMENTS: Xe PHYSISORBED ON Ag(111)}

To demonstrate the capabilities of our chamber, we chose xenon physisorbed on the $\mathrm{Ag}$ (111) surface for several reasons. Webb and co-workers ${ }^{13}$ have shown the $\mathrm{Ag}$ (111) surface to be of prototypical quality in physisorption studies of rare gases. The surface is exceptionally smooth (small corrugation in the adatom-substrate potential), and there are well established procedures for its preparation. ${ }^{16,17}$ The large $\mathrm{x}$-ray scattering cross section of $\mathrm{Xe}$ makes it exceptionally favorable for surface diffraction as demonstrated in previous synchrotron $\mathrm{x}$-ray experiments on the melting of a Xe monolayer on the $\mathrm{Ag}(111)$ surface. ${ }^{18}$ Our ultimate plan is to investigate the structure and phase transitions of the $\mathrm{Xe} / \mathrm{Ag}$ (111) system in regions of its phase diagram which are either inaccessible to LEED or which have not yet been explored by $\mathrm{x}$-ray scattering.

The experiments were conducted at the X18A beam line of the NSLS. The following procedure was used to prepare the sample. After mounting of the UHV chamber on the diffractometer, the sample is withdrawn to the preparation position (PP in Fig. 3). The shutter ( $S$, Figs. 2 and 3 ) is closed and the sample cleaned by Xe sputtering at 0.5 $\mathrm{keV}$ incident energy for $8 \mathrm{~h}$ at a pressure of $5.5 \times 10^{-5}$ Torr. ${ }^{17}$ The sample is then anncaled at $550^{\circ} \mathrm{C}$ for $30 \mathrm{~min}$. After translating the sample back to LEED/Auger/x-ray position, an Auger spectrum of the $\mathrm{Ag}(111)$ surface is 


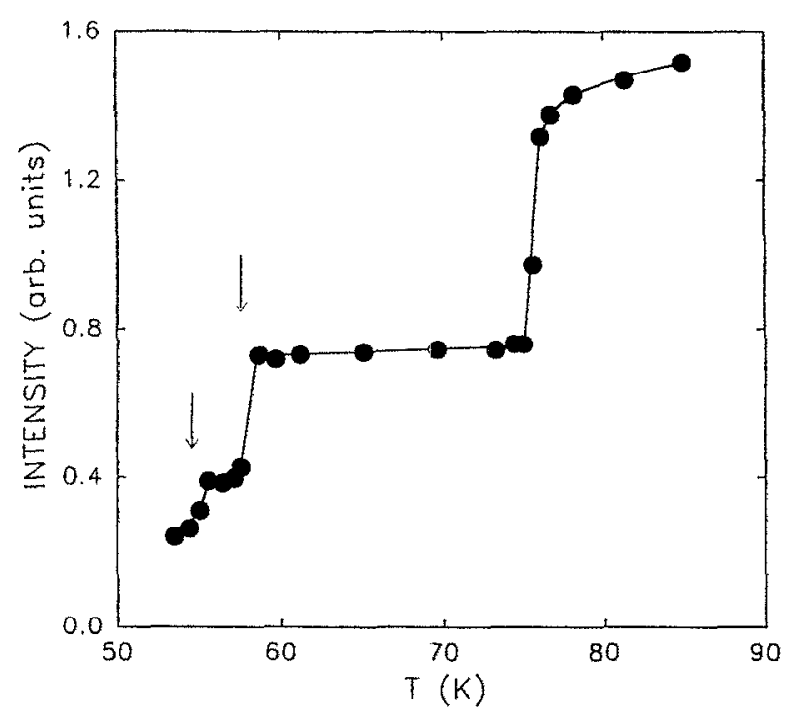

FIG. 5. The $\mathrm{Ag}(10)$ LEED spot intensity as a function of temperature for a fixed flux of gas incident on the sample: $P_{\text {eff }}=8.3 \times 10^{-8}$ Torr as defined in the text. The solid line is a guide to the eye. Arrows mark completion of the first layer at $T=58.1 \mathrm{~K}$ and of the second layer at $T=55.3 \mathrm{~K}$.

taken. This treatment is repeated until there are no detectable impurities on the surface.

To begin the $x$-ray experiment, the sample is centered in the x-ray beam which is focused by a platinum-coated mirror to a size typically $3 \mathrm{~mm}$ horizontally by $2 \mathrm{~mm}$ vertically. The sample's orientation was determined crystallographically by observing three $\mathrm{Ag}$ bulk reflections ${ }^{10.11}$ using an $x$-ray wavelength of $1.20 \AA$ as described in Sec. II. Alignment was checked by scanning through $\mathrm{Ag}(10)$ crystal truncation rod $^{19}$ at different fixed values of the momentum transfer perpendicular to the surface, $\mathbf{Q}_{\perp}$.

The Xe film is prepared by introducing $\mathrm{Xe}$ gas (99.995\% minimum purity) through a leak valve into the dosing tube situated in front of the sample. The background pressure in the chamber is typically adjusted to $\sim 1.0 \times 10^{-7}$ Torr. This yields an effective Xe pressure at the surface $P_{\text {eff }}=8.3 \times 10^{-8}$ Torr which is determined as described below. The substrate is initially at a temperature of $\sim 100 \mathrm{~K}$ which is then reduced while monitoring the $\mathrm{Ag}(10)$ LEED spot intensity. During this time, the $\mathrm{x}$-ray beam is turned off to avoid saturation of the electron multiplier (microchannel plates).

The $\mathrm{Ag}(10)$ LEED spot intensity as a function of temperature is shown in Fig. 5. The first sharp drop in intensity is attributed to the condensation of a Xe monolayer while the second step corresponds to bilayer formation. ${ }^{13}$ Temperatures at the completion of the monolaycr and bilayer are indicated by arrows in Fig. 5 . We were unable to resolve steps corresponding to the third and higher $\mathrm{Xe}$ layers The temperature of the last step is associated with the nucleation of solid bulk Xe on the surface which is at equilibrium with the gas from the dosing tube. This temperature can be used to determine the effective pressure of the dosing gas. ${ }^{13}$

Upon monolayer completion, the LEED system is
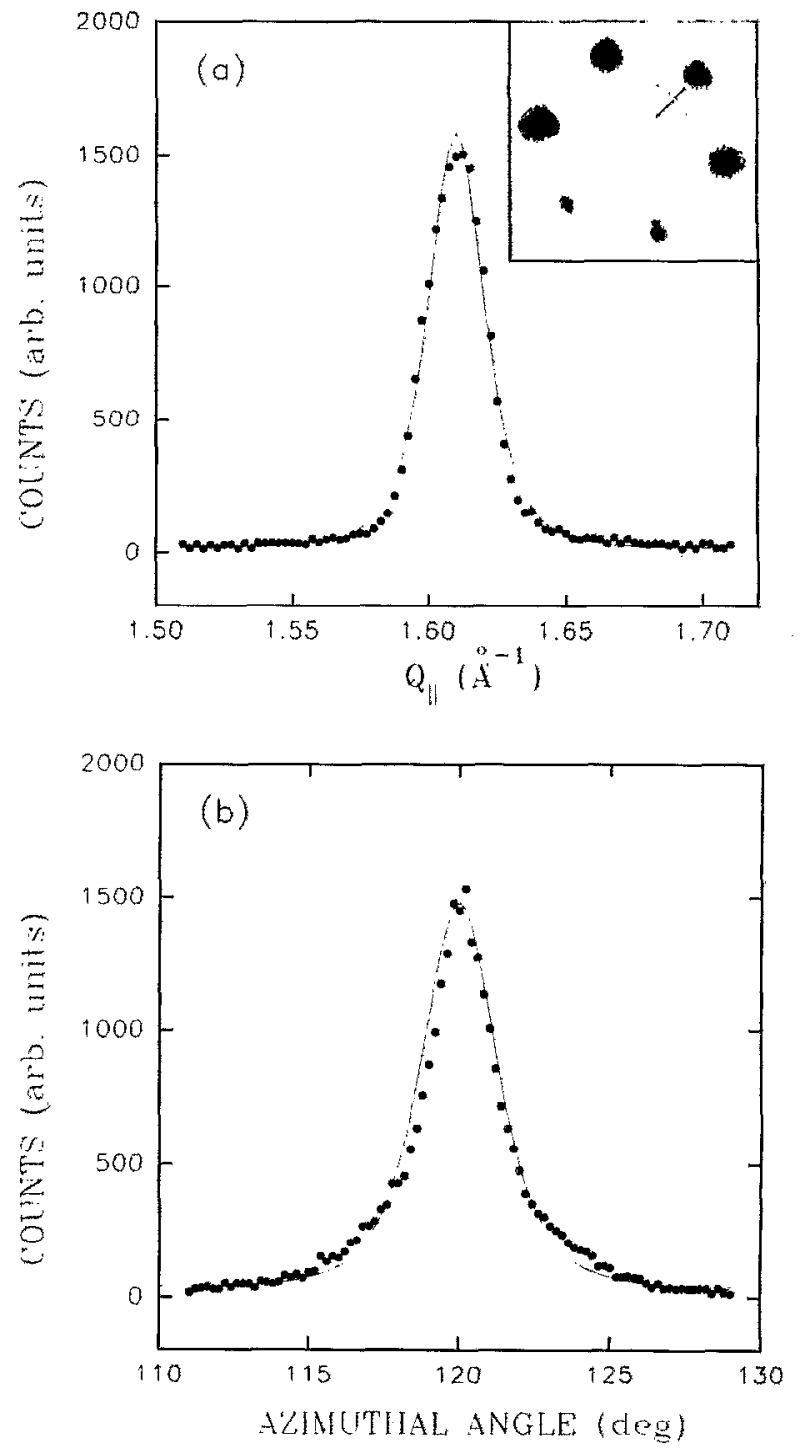

FIG. 6. X-ray diffraction scans [at fixed $Q_{1}=0.2 \AA^{-1}$ ] from a Xe monolayer physisorbed on the $\mathrm{Ag}(111)$ surface: (a) radial scan and (b) transverse scan. The solid curves through the data are best fits to a Lorentziansquared functional form (Refs. 18 and 19). Inset in (a) shows a LEED pattern obtained with this system with $\mathrm{x}$-ray scan directions superimposed: solid line for the radial scan and dashed line for the transverse scan.

turned off and the $x$-ray beam now of wavelength $1.72 \AA$ is opened for a precision determination of the Xe-Xe spacing in the film. Figure 6 shows typical radial and transverse $\mathrm{x}$-ray scans through a $\mathrm{Xe}(10)$ rod at monolayer coverage. The $x$-ray beam is incident upon the sample at an angle $\alpha=1.5^{\circ}$ [Fig. 1(b)]. At monolayer coverage, the counting rate is about 2200 counts/min from the film with a background rate of 50 counts/min. From the radial scan the Xe-Xe spacing of the film can be determined with an accuracy of $\sim 0.5 \%$. This uncertainty is determined primarily by errors in aligning the incident beam and the sample at the Huber center. Changes in the Xe-Xe spacing can be measured with a relative precision of $0.02 \%$.

The coherence length of the film and the adsorbing surface can be determined after deconvolution of the spec- 
trometer resolution from the radial scan. The FWHM of the peak in the radial scan is $0.02 \AA^{-1}$ with a resolution of $0.01 \AA^{-1}$ (FWHM) as determined by a slit in front of the detector. This yields a coherence length of the xenon monolayer of $\sim 300 \AA$. A similar scan of the bare $\mathrm{Ag}(111)$ surface gives a coherence length of $\sim 900 \AA$. The FWHM of the transverse scan in Fig. $6(\mathrm{~b})$ is about $3^{\circ}$ with the resolution of $0.03^{\circ}$. The large azimuthal width of the $\mathrm{Xe}(10)$ rod was also reported in Ref. 18. Its origin is not known with certainty, but it may be related to imperfect domain growth of the $\mathrm{Xe}$ monolayer from surface steps. ${ }^{13,20}$

With the completion of the linear drive for the detector described above, we expect to be able to perform specular $\mathrm{x}$-ray scans as well as scans along the $\mathrm{Xe}(10)$ rod. Such out-of-plane scans should allow determination of the film thickness above two layers, a coverage range which is inaccessible to LEED. We note that similar studies have already been conducted with a rotating anode source with $X e$ adsorbed on the graphite (0001) surface by Hong and Birgeneau. ${ }^{21}$ However, these experiments used a vermicular graphite ballast for the Xe coverage determination. The authors concluded that their interpretation of incomplete wetting of $\mathrm{Xe}$ on graphite at low temperatures may be complicated by capillary condensation effects in the graphite ballast. The in situ LEED capability in our chamber used for Xe coverage determination below three layers should allow us to perform experiments of this type without a ballast.

\section{SUMMARY}

We have described a transportable UHV chamber for use in surface $\mathrm{x}$-ray diffraction providing a combination of in situ LEED and low-temperature capabilities which has not been available in previous chambers. Moreover, the chamber is mountable on a standard x-ray diffractometer. The utility of these features for studies of the structure and phase transitions of physisorbed films has been demonstrated in some preliminary investigations of the $\mathrm{Xe} /$ $\mathrm{Ag}(111)$ system.

\section{ACKNOWLEDGMENTS}

We are indebted to a number of people for contributing to the design and construction of our chamber. P. Zschack and $Y$. W. Chung shared their experiences in designing the first MATRIX surface chamber while P. Stair and A. Test- oni were of particular help in the our LEED system design. J. Z. Larese provided advice, support facilities, and laboratory space during a year's stay of S.-K. W., P. D., and H. T. at Brookhaven National Laboratory. C. Holmes, S. Potts, W. Stiefermann, and D. Valleroy of the Physics Department Machine Shop, University of Missouri-Columbia provided invaluable assistance in both design and construction throughout this project. We thank K. W. Herwig for a critical reading of the manuscript. This research was supported by U. S. NSF Grant Nos. DMR-8304366, DMR-8704938, and DMR-9011069 (H. T.), U. S. Department of Energy Grant No. DE-FG02-85ER45183 of the MATRIX Participating Research Team (S. N. E. and H. T.), University of Missouri Multicampus Weldon Springs Awards (H. T.), and a Research Council Grant of the University of Missouri-Columbia (H. T.).

${ }^{1}$ R. Feidenhans'l, Surf. Sci. Rep. 10, 105 (1989).

${ }^{2}$ S. Brennan and P. Eisenberger, Nucl. Instrum. Methods A 222, 164 (1984).

${ }^{3}$ P. H. Fuoss and I. K. Robinson, Nucl. Instrum. Methods A 222, 171 (1984).

${ }^{4}$ E. Vlieg, A. van't Ent, A. P. De Jongh, H. Neerings, and J. F. van der Veen, Nucl. Instrum. Methods A 262, 522 (1987).

${ }^{5}$ P. Zschack, J. B. Cohen, and Y. W. Chung, J. Appl. Crystallogr. 21, 466 (1988)

${ }^{6}$ P. Zschack, Ph. D. thesis, Northwestern University, 1989 (unpublished).

${ }^{7}$ Midwest Analytical Team for Research Instrumentation of X Rays.

${ }^{8}$ W. C. Marra, Ph. D. thesis, Stevens Institute of Technology, 1981 (unpublished); R. Feidenhans"l, Ph.D. thesis, Ris $\phi$ National Laboratory, 1986, unpublished as cited in Ref. 1.

${ }^{9}$ The Be window was fabricated and electron beam welded to the stainless steel chamber by Electrofusion Corp. The Be used was Commercial Grade S200E Type I with the main contaminant $0.09 \mathrm{wt} . \% \mathrm{Fe}$, resulting in a transmission of $78 \%$ at a wavelength of $1.5 \AA$.

${ }^{10}$ S. K. Wang, Ph.D. thesis, University of Missouri-Columbia, 1992 (unpublished).

${ }^{11}$ S.-K. Wang and P. Dai (unpublished).

${ }^{12}$ E. Vlieg, J. F. van der Veen, J. E. Macdonald, and M. Miller, J Appl Crystallogr. 20, 330 (1987).

${ }^{13}$ J. Unguris, L. W. Bruch, E. R. Moog, and M. B. Webb, Surf. Sci. 87, 415 (1979).

${ }^{14}$ P. C. Stair, Rev. Sci. Instrum. 51, 132 (1980).

${ }^{15}$ M. D. Chinn and S. C. Fain, Jr., J. Vac. Sci. Technol. 14, 314 (1977).

${ }^{16}$ E. R. Jones, J. T. McKinney, and M. B. Webb, Phys. Rev. 151, 476 (1966).

${ }^{17}$ G. McElhiney, H. Papp, and J. Pritchard, Surf. Sci. 54, 617 (1976)

${ }^{18}$ N. Greiser, G. A. Held, R. Frahm, R. L. Greene, P. M. Horn, and R. M. Suter, Phys. Rev. Lett, 59, 1706 (1987).

${ }^{19}$ M. F. Toney, J. G. Gordon, M. G. Samant, G. L. Borges, O. R. Melroy, L.-S. Kau, D. G. Wiesler, D. Yee, and L. B. Sorensen, Phys. Rev. B 42, 5594 (1990)

${ }^{20}$ P. I. Cohen, J. Unguris, and M. B. Webb, Surf. Sci. 58, 429 (1976).

${ }^{21} \mathrm{H}$. Hong and R. J. Birgeneau, Z. Phys. B 77, 413 (1989). 